\title{
FINITE ELEMENT ANALYSIS OF PULSED LASER BEAM INDUCED TEMPERATURE PROFILES IN MAGNETO-OPTIC RECORDING THIN FILMS
}

\author{
Seh Kwang LEE and Soon Gwang KIM \\ Division of Materials Science and Engineering, Korea Advanced Institute of Science and Technology, \\ P. O. Box 131, Cheongryang, Seoul, Korea
}

\section{ABSTRACT}

Variation of temperature profiles during the magneto-optic recording process in monolayered $\mathrm{TbFe}$ and GdCo thin film was analyzed by the two-dimensional finite element method. The effects of laser power and film thickness on the heating behavior of the film were mainly examined as the major parameters to determine the recorded bit size. It has been shown that maximum local temperature rise on the surface of film at the beam center can be estimated by a simple relation to the film thickness.

\section{INTRODUCTION}

When a thin film material is considered for use as the active layer of an optical storage medium, the response of the film upon the irradiation of pulsed laser beam is of utmost importance. In magneto-optical recording where bits are formed or erased thermomagnetically,i.e., reversal of magnetic polarity at the locally heated region of film utilizing the coercivity drop as the result of laser heating[1], understanding of major controlling factors on spatial and temporal distribution in thin film is particularly essential not only for the development of recording medium but also in the design of recording system[2]. Various material parameters such as thermal conductivity, reflectivity, specific heat, absorption coefficient, film thickness, substrate properties combined with laser irradiation conditions (output power, pulse duration) must be taken into account in the prediction and their relative effects have to be estimated quantitatively[3], [4], [5] .

In this work, the finite element method was used to analyze the static two-dimensional transient heating and cooling characteristics of rare earth-transition metal films ( $\mathrm{TbFe}$ and GdCo) having monolayered structure as a simplified picture. The effects of laser power and film thickness on the heating behavior of the film were mainly examined as the major parameters to determine the bit size.

\section{MODEL FOR LASER BEAM ENERGY TRANSFER}

When a Gaussian laser beam of incident power P irradiates the surface of thin film as shown in Fig.l, the beam is partially reflected and the rest is transmitted into the film. Assuming that the laser beam with circular cross section impinge on the stationary film surface, the temperature distribution is axisymmetric with respect to the beam center. The heat transfer model can therefore be most conveniently described by adopting a cylindrical coordinate system which enables the treatment simpler by reducing it to two dimensional model.

The Gaussian intensity profile of the beam, I $(r, z)$, at the focal point $z=0$ of the objective lens, is given by $\mathrm{Eq} \cdot(1)$,

$$
I(r, 0)=I_{0} \exp \left(-\left(r / r_{0}\right)^{2}\right)
$$

whene $r$ is the radial distance from the beam center at which the intensity drops by a factor e from the maximum value at the center of the heam, and $I_{\text {is }}$ is the peak intensity which is given by $\mathrm{P} / \pi \mathrm{r}^{2}$. The source power density, $S(r, z, t)$, absorbed in the film can best be described by Eq. (2) $[4],[5]$;

$$
S(r, z, t)=(1-R) M(t) I_{0} \exp \left(-\left(r / r_{0}\right)^{2}\right) \alpha \exp (-\alpha z)
$$

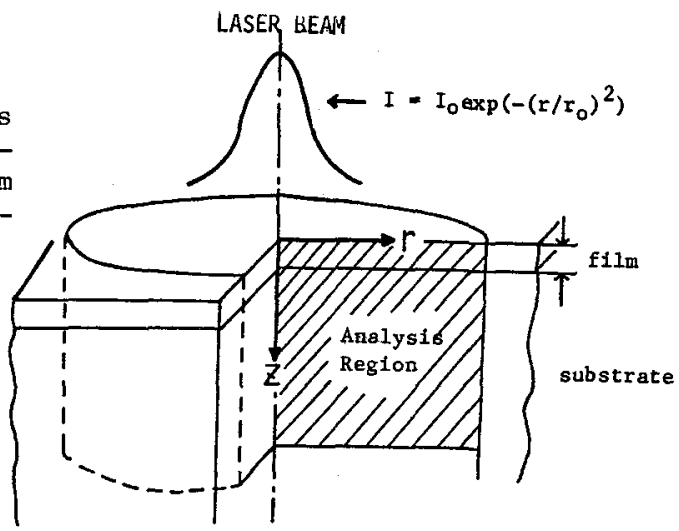

where $R$ and $\alpha$ are reflectivity and absorption coefficient, respectively, and $M(t)$ is the modulation function of the laser power as shown schematically in Fig.2.

For metallic materials, however, the absorption coefficient is generally so high the light energy absorption may reasonably be assumed to occur at the surface of the film. For example, the penetration depth of laser beam into the rare earth-transition metal (RE-TM) alloy can be estimated to be about $100 \AA$ assuming its absorption coefficient $10 \%$ [4]. Eq. (2) can then be approximated to a more simplified form as $\mathrm{Eq} .(3)$;

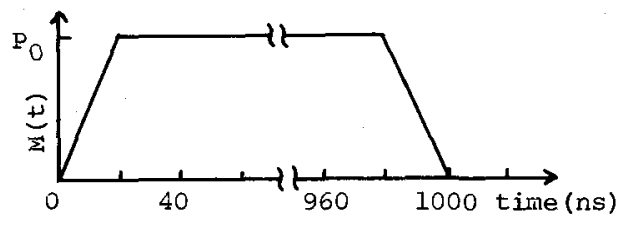

Fig.2. The modulation function of the laser power. 


$$
S(r, t)=(1-R) M(t) I_{0} \exp \left(-\left(r / r_{0}\right)^{2}\right)
$$

In order to find the temperature distribution of a film, we must solve the following heat diffusion equation;

$$
c \rho \frac{\partial T}{\partial t}=k \nabla^{2} T+s(r, t)
$$

where $\rho$ is the mass dersity, $c$ is the specific heat, $k$ is the thermal conductivity and $S(r, t)$ is the source power density given by Eq. (3). The convectional heat loss was neglected in Eq. (4).

\section{FINITE ELEMENT FORMULATION}

For the development of a finite element solution scheme for Eq. (4), a variational formulation can be employed[6]. The original differential equation is then transformed into the matrix equation. Detailed procedures may be found elsewhere[6]. In this calculation, the laser beam radius was fixed as $0.6 \mu \mathrm{m}$ and film thickness was assumed to be $1000 \AA$ except the case of study on the effect of variation. The magnetic film and substrate were subdivided into 8 layers, 160 volume elements as shown in Fig.3. The heat source density of each nodal point at the top surface of film was calculated using Eq. (3). As a boundary condition, the initial temperatures of all nodal points were assigned to room temperature, and temperatures of the boundaries $(r=2$. O $\mu \mathrm{m}$ and $\mathrm{z}=1.5 \mu \mathrm{m})$ were assumed to retain room temperature during the heating and cooling cycle. For the air/film interface, air was assumed to be an insulator because of very low thermal conductivity. The continuity of temperature and heat flux at the film/substrate interface were also assumed in this calculation. Temperature of each nodal point was cal- Fig.3. Details of finite element grid culated by a well-established computer program ADINAT[7] for used in this calculation. First two every time step period. The time step period was chosen as a layers represent thin film and the compromise to ensure reasonable calculation accuracy with mi- last six layers correspond to substnimum computing time; i.e.,10nsec for initial $100 \mathrm{nsec}$ includ- rate.

ing the pulse rise stage, 50nsec between 100 and $950 \mathrm{nsec}$, and then $10 \mathrm{nsec}$ after $950 \mathrm{nsec}$ for $100 \mathrm{nsec}$ including the pulse fall stage.

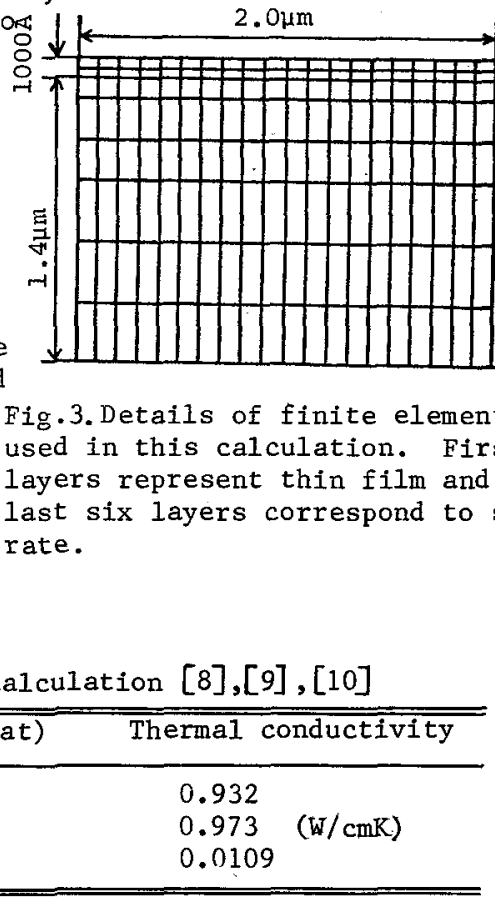

The optical and thermal constants for film and substrate materials used in this calculation are listed in Table 1. The specific heat of the amorphous TbFe and GdCo alloy was derived indirectly from the corresponding values of the constituent elements by using the Neumann-Kopps' rule[9], and the density for them was calculated from the Miedema's theory[10].

\section{RESULTS AND DISCUSSION}

In order to record a bit on a TbFe film statically, the film should be heated up by laser irradiation over $100^{\circ} \mathrm{C}$ where the coercivity drops to several hundred Oersted, and the bias field which is larger than the coercivity of $\mathrm{TbFe}$ at this elevated temperature is then applied to form a bit which corresponds to a circular area higher than $100^{\circ} \mathrm{C}$. In this regard, a radius of $100^{\circ} \mathrm{C}$ isotherm may be defined as a measure of the bit size and the investigation of its behavior is our major work.

Here the temperature profile was calculated by the numerical method for the RE-TM film on glass substrate upon irradiation by a $10-\mathrm{mW}, 820 \mathrm{~nm}$ AlGaAs laser with pulse duration of $980 \mathrm{nsec}$. Fig.4 shows the radial temperature distribution for a heating ( $t=0-980 \mathrm{~ns}$ ) and cooling ( $t=980-1250 \mathrm{~ns}$ ) cycle in $\mathrm{TbFe}\left(\mathrm{Tb}_{24} \mathrm{Fe}_{76}\right.$ ) film on glass. And Fig.5 shows the temperature vs, time curves at various radial positions on the top surface of TbFe film. The temperature initially rises steeply, but the heating rate gradually decreases after about $100 \mathrm{nsec}$, then approaches a constant value beyond $t \simeq$

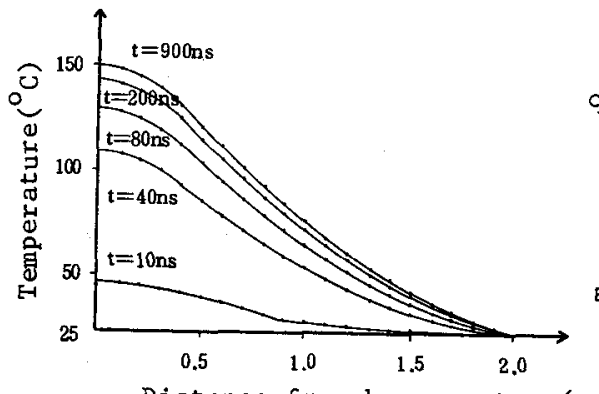

Distance from beain center $(\mu \mathrm{m})$

(a) heating cycle

Fig.4. Radial temperature distribution : film material=TbFe; film thickness $=1000 \AA$; substrate=glass ; laser power $=10 \mathrm{~mW}$; laser beam radius $=0.6 \mu \mathrm{m}$.

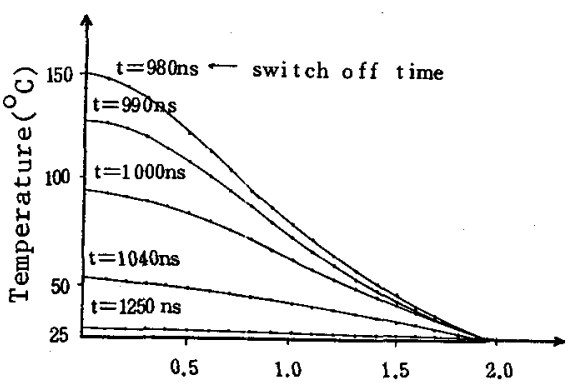

200 nsec until switch-off. The cooling takes place almost instantaneously down to about $50-70^{\circ} \mathrm{C}$, then slowly to room temperature. Because of the high thermal conductivity of $\mathrm{TbFe}$, temperature variation along the $z$ direction within the film was less than $5^{\circ} \mathrm{C}$. Calculation on GdC̣o film also revealed very similar heating and

Distance from beam center ( $\mu \mathrm{m}$ ) cooling behavior to that of (b) cooling cycle $\mathrm{TbFe}$; the maximum temperature of film being lower by only $7-8^{\circ} \mathrm{C}$ compared to the case of $\mathrm{TbFe}$. This also 


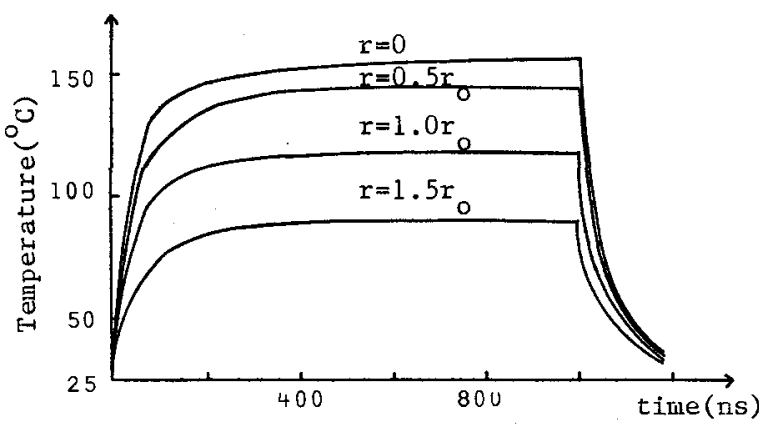

Fig.5. Temperature vs. time curves at various radial positions on the surface of TbFe film. other conditions are the same as, those in Fig. 4 .

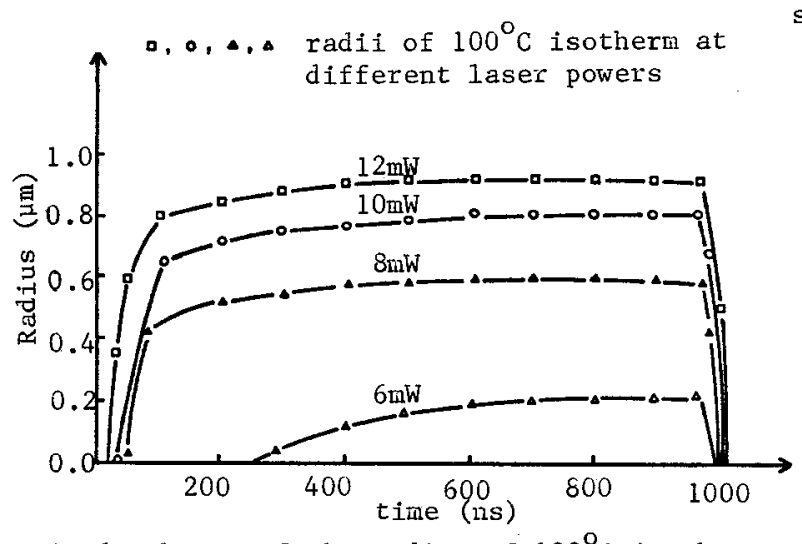

Fig.6. Change of the radius of $100^{\circ} \mathrm{C}$ isotherm of TbFe film at various laser power : pulse duration $=980 \mathrm{nsec}$; film thickness $=1000 \AA$. implies that change of thermal conductivity and specific heat due to variation in chemical composition of $\mathrm{RE}-\mathrm{TM}$ alloy does not affect temperature profile significantly.

For a given substrate material, major factors which influence the temperature profile of monolayered RE-TM films are the power of focused laser beam and film thickness[4],[5]. Fig.6 illustrates the effect of laser power on the radial expansion of $100^{\circ} \mathrm{C}$ isotherm during pulsed laser irradiation. The radius of $100^{\circ} \mathrm{C}$ isotherm increases explosively within $100 \mathrm{nsec}$ after that it tends to be a constant value. Fig. 6 provides a valuable criterion in the design of optical disk system because it either determines proper laser power and pulse duration to obtain a desired bit size or shows the achievable bit size for a given laser power. For instance, if the pulse duration time is set to $500 \mathrm{nsec}$, the laser power of $7.5 \mathrm{riw}$ for recording lum-diameter bit may be obtained from the laser power vs. radius of $100^{\circ} \mathrm{C}$ isothermal curve as shown in Fig. 7 .

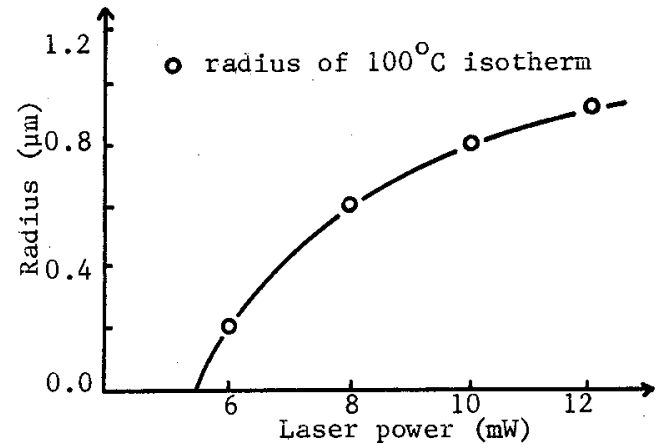

Fig.7. The radius of $100^{\circ} \mathrm{C}$ isotherm vs. laser power for $500 \mathrm{nsec}$ pulse duration. The open circles denote the calculated values, solid line is an interpolated curve.

In order to find the effect of film thickness on the temperature profile, calculation was carried out for a range of film thickness from $400 \AA$ to $1200 \AA$ increasing by every $200 \AA$. The effect of film thickness may be represented in terms of the variation of maximum local temperature rise at the beam center on power-off time as shown in Fig.8. It is expected that maximum local temperature increases markedly with decreasing film thickness. A least squares fitting showed that the curve can be expressed by the following equation within $1^{\circ} \mathrm{C}$ accuracy;

$$
\Delta \mathrm{T}=\mathrm{T}_{\max }-\mathrm{T}_{\text {room }}=\frac{1}{7.05 \times 10^{-2} \mathrm{~d}+6.27 \times 10^{-4}}
$$

where $\mathrm{d}$ is the film thickness in $\mu \mathrm{m}$. The usefulness of Eq. (5) in the design of layer structure for magneto-optic disk is evident and may be more comprehensive if the physical meaning of Eq. (5) be understood by following arguments described below.

The fact that the film temperature at the beam center $(r=0)$ is maximum at any given depth leads to $\partial T / \partial r=0$. This simplifies the problem of through-thickness heat transfer at the beam center to a one-dimensional one as schematical1y illustrated in Fig.9. For convenience, let the film/substrate interface be located at $z=0$. Since the film $(-d<z<0)$ has much higher thermal

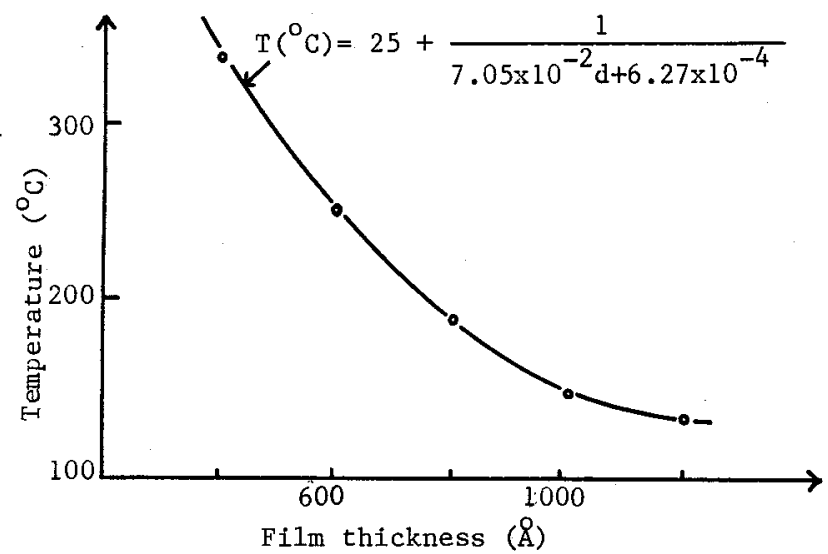

Fig.8. The effect of variation in film thickness for TbFe alloy. The open circles are calculated values by the finite element method whereas the solid line is a calculated curve by using Eq. (5). conductivity than the substrate $(0<z), \Delta T$ of the film may reasonably be assumed to be uniform across the thickness, whereas $\Delta T$ of the substrate decreases with increasing $z . \Delta T$ vs. $z$ curve at $z>0$ can be obtained by solving the following one-dimensional heat diffusion equation ;

$$
\rho_{s} c \frac{\partial T}{\partial t}=k \frac{\partial^{2} T}{\partial z^{2}}
$$

where the subscript $s$ denotes substrate. At the instance of power-off at which the temperature of film reaches the maximum, $\partial \mathrm{T} / \partial \mathrm{t}$ is constant as evident in Fig.5, and independent of the thickness [11]. Eq.(6) can then be simplified as Eq.(7); 


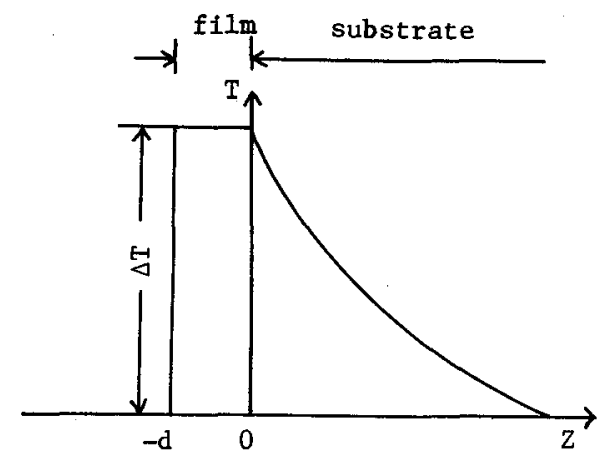

Fig.9 Schematic diagram of one-dimensional temperature distribution along $z$ direction at the beam center.

$$
\frac{\partial^{2} T}{\partial z^{2}}-\frac{\rho s^{c} s}{k_{s}} \beta T=0
$$

where $\beta$ is the constant value of $\partial \mathrm{T} / \partial \mathrm{t}$. Since $\mathrm{T}=\Delta \mathrm{T}$ at $z=0$, the solution of Eq.(7) is given as Eq. (8) ;

$$
\mathrm{T}(\mathrm{z})=\Delta \mathrm{T} \exp (-\mathrm{qz})
$$

where $q$ is $\left(\rho_{c} \beta / k_{c}\right)^{1 / 2}$. Total heat, $H$, absorbed by the unit area of the film at the beam center can be expressed by the equality of $\mathrm{Eq} \cdot(9)$;

$$
\mathrm{H}=\rho_{f} c_{f} d \Delta T+\int_{0}^{\infty} \Delta T \exp (-q z) d z
$$

where $p_{f} c_{f}$ are the density and the specific heat of the film, respectively, which are constants. Since both the integration term on the right hand and $\mathrm{H}$ are constants, it leads to Eq.(10), which has an equivalent form as $\mathrm{Eq} \cdot(5)$;

$$
\Delta \mathrm{T}=\frac{1}{\mathrm{ad}+\mathrm{b}}, \mathrm{a}=\rho_{\mathrm{f}} \mathrm{c}_{\mathrm{f}} / \mathrm{H}, \mathrm{b}=1 / \mathrm{Hq}
$$

where $a$ and $b$ are constants which can be directly calculated from thermal and optical properties of film and substrate, and the laser irradiation condition. And the value bf $\beta$ should be obtained from temperature vs. time curve for calculating b. In Eq.(10), a is a constant related only to the properties of thin film and $b$ to the properties of substrate. Although this equation is strictly applicable only for the case of films whose thickness is small enough so that the temperature gradient across the thickness can be ignored, and also in spite of the difficulty in determining the accurate value of $\beta$, it provides a valuable tool in determining proper thickness of film since the curve obtained from Eq.(5) allows extrapolation for a wide range of thickness if the calculations are made for only a few thickness values.

\section{CONCLUSION}

Calculation of temperature profile in RE-TM thin films by using a two-dimensional finite element method indicated that the radius of $100^{\circ} \mathrm{C}$ isotherm as a measure of the recorded bit size was dependent on the composition of the film material only slightly and mainly dependent on laser power and film thickness. A simple relationship between the maximum heating temperature by laser beam irradiation and the film thickness was derived empirically. Interpretation of the physical meaning for the empirical relation was made by applying a one-dimensional heat transfer model on a given film/ substrate system.

\section{REFERENCES}

1. S. Honda, K. Ueda, T. Kusuda : J. App1. Phys. 52 (1981) 2295.

2. M. Mansuripur, G.A.N. Conne11 : J. Appl. Phys. 54 (1983) 4794.

3. M. Mansuripur, G.A.N. Connell, J.W. Goodman : Appl. Opt. 21 (1982) 1106.

4. M.K. Bhattacharyya, Z.J. Cendes : J. Appl. Phys. 57 (1985) 3894.

5. S.-C. Shin : J. Magn. Magn. Mat. 61 (1986) 301.

6. K.J. Bathe: "Finite Element Procedures in Engineering Analysis", Prentice-Hall Inc., New Jersey, (1982) P. 407

7. K.J. Bathe : "ADINAT User's manual", ADINA Eng. Inc. (1981).

8. R.C. Weast : "CRC Handbook of Chemistry and Physics", CRC Press, Florida (1980).

9. R.C. Swallin: "Thermodynamics of Solids", 2nd ed., John Wiley and Sons, Inc., New York (1972) P. 83 .

10. A.K. Niessen, F.R. de Boer, R. Boom, P.F. de Chatel, W.C.N. Mattens, A.R. Miedema : Calphad 7 (1983) 51 .

11. S.G. Kim, S.K. Lee, J.C. Park, C.J. Kim, KAIST Res. Rep. to MOST, N166-2591-1 (1986) 82. 